\title{
Effects Of Whole Body Vibration On Vertical Jump Performance Following Exercise Induced Muscle Damage
}

\author{
Nicole C. Dabbs (Corresponding author) \\ 1Department of Kinesiology, California State University, San Bernardino \\ 5500 University Parkway, HP 210, San Bernardino, CA 92407 \\ Tel: 1-(909) 537-7565 E-mail: ndabbs@csusb.edu \\ Lee E. Brown \\ 2Department of Kinesiology, California State University, Fullerton \\ 800 N. State College Blvd, Fullerton, CA 92833 \\ Tel: 1-657-278-4605 E-mail: leebrown@fullerton.edu \\ John C. Garner \\ 3Department of Health, Exercise Science, \& Recreation Management, The University of Mississippi \\ PO Box 1848, Turner 215. University, MS 38677 \\ Tel: 1-662-915-5526 E-mail: jcgarner@olemiss.edu
}

Received: 21-12- 2013

doi:10.7575/aiac.ijkss.v.2n.1p.23
Accepted: 18-01- 2014

Published: 31-01- 2014

URL: http://dx.doi.org/10.7575/aiac.ijkss.v.2n.1p.23

\begin{abstract}
Enhancing vertical jump performance is critical for many sports. Following high intensity training, individuals often experience exercise induced muscle damage (EIMD). Many recovery modalities have been tested with conflicting results. The purpose of this investigation was to determine the effect of whole-body vibration (WBV) on vertical jump performance following EIMD. 27 females volunteered for 7 sessions and were randomly assigned to a treatment or control group and administered each testing day. Vertical jump performance was assessed via vertical jump height (VJH), peak power output (PPO), rate of force development (RFD), relative ground reaction force (GRFz), and peak activation ratio of the vastus medialis (VM) via electromyography (EMG) before and after 3 days of EIMD via split squats. Two testing sets were collected each day, consisting of pre measures followed by WBV or control, and then post second measures. A 2x8 (group x time) mixed factor analysis of variance (ANOVA) was conducted for each variable. No significant interactions or group differences were found in any variable. Significant main effects for time were found in any variable, indicating performance declined following muscle damage. These results indicate that WBV does not aid in muscle recovery or vertical jump performance following EIMD.
\end{abstract}

Keywords: muscle recovery, muscle soreness, females

\section{Introduction}

Resistance training is a common exercise and training tool to increase muscular strength, hypertrophy and endurance. While resistance training is essential for enhancing performance, it can also lead to some temporary debilitations due to exercise induced muscle damage (EIMD). Delayed onset muscle soreness (DOMS) has been identified as a painful and undesirable side effects of EIMD (Schutzer \& Graves, 2004) and usually peaks at 24 to 72 hours (Gibson, ArendtNielsen, \& Graven-Nielsen, 2006; McHugh, Connolly, Eston, \& Gleim, 1999). EIMD presents as tenderness, pain, swelling, and muscle stiffness (McHugh et al., 1999) and it has been suggested that these symptoms are related to an inflammatory process. This is based on a lack of evidence of neural inhibition of damaged muscle (McHugh et al., 1999) or changes in motor unit activation (Sayers, Knight, Clarkson, Van Wegen, \& Kamen, 2001). It has been well documented that EIMD occurs from repeated eccentric muscle actions, resulting in decreased force production (Armstrong, Warren, \& Warren, 1991; Proske \& Morgan, 2001). Evidence of disruption of the normal alignment of skeletal muscle and disruption of the z-lines of sarcomeres has been seen in damaged musculature (Friden, Sjostrom, \& Ekblom, 1983; Stauber, Clarkson, Fritz, \& Evans, 1990). Muscle soreness has been shown to occur initially from the inflammatory process with production of prostaglandin $\mathrm{E}_{2}$ observed at 24, 48, and 72 hours (Friden et al., 1983) which sensitizes type III and IV afferent fibers of muscle connective tissue, which are highly correlated with DOMS (Connolly, Sayers, \& McHugh, 2003).

During most performance activities, the main goal is to maximize power output; however, with EIMD, power generation may be compromised. Previous work has demonstrated that peak power output is immediately reduced following eccentric muscle actions in the knee extensors during isokinetic cycling (Sargeant \& Dolan, 1987) and a Wingate cycle test (Byrne \& Eston, 2002a) while continuing to decrease up to 2 days post exercise. A decrease in 
power output has also been shown during intermittent maximal sprints on a cycle ergometer after 10 sets of 10 plyometric jumps to induce muscle damage (Twist \& Eston, 2005). Further, vertical jump performance is related to peak power output and has found to be compromised following EIMD. Studies have found a prolonged reduction in maximal force production, EMG activity, ground reaction force, stretch reflex sensitivity, muscle and joint stiffness regulation and the stretch shortening cycle (Avela \& Komi, 1998; Horita, Komi, Nicol, \& Kyrolainen, 1999) following EIMD; which all play a role in jumping performance. Vertical jump performance with and without a countermovement have been shown to have immediate and long-lasting reductions in performance up to 4 days post exercise but are dependent on jump type (Byrne \& Eston, 2002b). Squat jumps have the most prolonged reduction in jump height compared to countermovement jumps and depth jumps.

Eccentric exercises are commonly used as a component of resistance training programs and have been shown to elicit DOMS, potentially causing reduction in sport performance. Previous research has studied several ways to control or prevent symptoms of EIMD (Cheung, Hume, \& Maxwell, 2003). Most current modalities have not been shown to be consistently effective, including, but are not limited to massage, cryotherapy, stretching, homeopathy, ultrasound, and electrical current (Cheung et al., 2003). Recently, WBV has been suggested as a novel modality to reduce or control symptoms of EIMD (Aminian-Far, Hadian, Olyaei, Talebian, \& Bakhtiary, 2011; Bakhtiary, Safavi-Farokhi, \& Aminian-Far, 2007; Rhea, Bunker, Marin, \& Lunt, 2009). Bakhtiary et al. 2007 found that WBV prior to eccentric loading might attenuate DOMS via possible mechanisms of increased blood flow to facilitate recovery and muscle regeneration or possible pain inhibition (Bakhtiary et al., 2007). Rhea et al. 2009, implemented WBV in combination with stretching and massage after strenuous exercise over a period of 72 hours and showed decreased pain perception in the WBV group (Rhea et al., 2009). Aminan-Far et al. 2011 also showed a reduction in DOMS symptoms and maximal isometric and isokinetic voluntary strength loss, creatine kinase, pain threshold and muscle soreness with WBV performed prior to eccentric exercises (Aminian-Far et al., 2011). However, to our knowledge, no study has investigated the effects of WBV following exercise induced muscle damage in vertical jump performance measures. Therefore, the purpose of this investigation was to determine the effects of WBV on jumping performance following exercise induced muscle damage in women.

\section{Methods}

The aim of the current study was to investigate acute and chronic effects of WBV exposure on jumping performance following EIMD. Therefore, we used a mixed factor design testing a control and WBV group's vertical jumping performance prior to muscle damage and for 3 days following EIMD.

\subsection{Participants}

Twenty-seven recreationally trained females (age $21 \pm 2$ yrs, height $172.38 \pm 92.27 \mathrm{~cm}$, mass $58.67 \pm 11.53 \mathrm{~kg}$ ) volunteered to participate in a 7-session protocol and provided written informed consent that was approved by the University's Institutional Review Board. Recreationally trained individuals were defined as meeting American College of Sports Medicine recommendations for healthy living and did not exceed 5 lower body workouts a week on a regular basis in the last 6 months. Participants with a recent history of lower body musculoskeletal or orthopedic injury or those taking medications that might alter balance, musculoskeletal system, or central nervous system functions relating to posture and motor control were excluded from participation. Additionally, individuals taking prescription pain and/or psychiatric medications were excluded. All participants were screened by questionaire for potential risk factors to the exercise protocol (e.g. rhabdomyolysis, bruising easily, etc.). They were asked not to perform any lower body exercise or take any pain medications 48 hours prior to testing sessions and during all testing days and to keep all food and water intake consistent throughout the study. The requests were verified through the use of a diet and phyical acitivity recall questionnaire completed by the participants. Furthermore, partipants were not scheduled for testing during their menstral cycle in order to avoid non-compliance with the instructions above.

\subsection{Measures}

\subsubsection{Vertical Jump}

Vertical jump performance was assessed on each visit using a combination of a Vertec ${ }^{\circ}$ (Sports Imports, Columbus, OH, USA) device and a Bertec ( Bertec Corp. Columbus, OH, USA) force platform sampling at 1080 Hz. Participants were instructed to perform three maximal countermovement vertical jumps (CMVJ; $15 \mathrm{~s}$ rest), with arm swing and were instructed to jump as quickly and high as possible. The Vertec was used as a visual target where participants could hit tabs indicating jump height. Vertical jump height was calculated by the difference between standing reach and maximum jump reach while peak power output (PPO) was calculated via the Sayers Equation (Sayers, Harackiewicz, Harman, Frykman, \& Rosenstein, 1999). Relative peak propulsion ground reaction force (GRFz) was calculated from peak z-force subtracted by body mass $(\mathrm{N} \square \mathrm{kg}$ ). Rate of force development (RFD) was derived from $\Delta$ Force/ $\Delta$ Time over the first $200 \mathrm{~ms}$ of the concentric phase of the vertical jump, beginning when force returned to body mass for the upward motion of the jump.

\subsubsection{Electromyography}

Bipolar surface electromyography (EMG) was recorded during maximal isometric voluntary contraction (MVIC) and maximal vertical jump on the left leg during each visit. Noraxon single electrodes (Noraxon USA Inc., Scottsdale, AZ, USA) were placed 3-5 cm apart with a ground electrode on the head of the tibia. Proper preparation included abrasion of the skin around the electrode site followed by cleansing with an alcohol swab. Data were recorded from the left vastus medialis (VM) using a Noraxon Telemyo 8-channel EMG system (Noraxon USA Inc., Scottsdale, AZ, USA) 
with a hardware band pass filter $(10-500 \mathrm{~Hz})$. During isometric MVIC, EMG signals were recorded for $5 \mathrm{~s}$ on a modified knee-extension machine (Body Solid; model GLCE-365; Forest Park, IL). Participants were seated with their hips at $90^{\circ}$ of flexion and with their knees fixed at a flexed position at a $60^{\circ}$ angle below horizontal. A strap was used to secure their left ankle to the lever arm. Ratios were calculated by dividing vertical jump peak EMG by MVIC peak EMG.

\subsubsection{DOMS Visual Analog Scale}

To assess soreness in the quadriceps participants were asked to rate the intensity of their pain/hurt/soreness in their quadriceps during a body weight squat. A $100 \mathrm{~mm}$ visual analog scale (VAS) was used to assess soreness. Participants were instructed to place a mark along the $100 \mathrm{~mm}$ line that corresponded to their intensity of pain. Anchors of "no pain" and "worst pain imaginable" were placed on the left and right end of the $100 \mathrm{~mm}$ line, respectively. Soreness was determined by measuring the interaction of the horizontal scale and the vertical line with a tape measure.

\subsection{Procedures}

During three familiarization sessions, participants read and signed the informed consent, filled out screening questionnaires, were measured for anthropometrics and performed all testing protocols. Following these familiarization sessions, participants visited the laboratory on 4 consecutive days and were randomly assigned to a control or treatment (WBV) group. Prior to each pre- measurement, all participants performed 2 sets of 15 meters of dynamic warm-ups including: jogs, gait swings, high knees, exaggerated lunges and Frankensteins. They were then assessed for pre-values on vertical jump performance, MVIC EMG and quadriceps muscle soreness. After pretesting, they performed an EIMD protocol, which consisted of split squats using a Jones Machine (Light Commercial model, Body Craft, Sunbury, OH) by performing 4 sets to momentary failure on each leg with a one-minute rest between sets. The Jones Machine was front loaded with $40 \%$ of their body mass. During split squats, the rested back leg was placed on a bench for support while the forward knee was flexed at 90 -degrees, allowing focus on single leg performance of the front leg. There were assisted on the concentric phase after they reached 90 degrees of front knee flexion in order to focus on the eccentric phase.

Immediately following the EIMD protocol, the control group performed 2 sets of body weight quarter squats on a flat surface for 30s with a 1:1 work to rest ratio. The WBV group performed 2 sets of body weight quarter squats on the vibration plate. An Power Plate (AIRdaptive 5, Performance Health Systems, Northbrook, IL) system was utilized to provide tri-axial vibration exposure. Vibration frequency was set at $30 \mathrm{~Hz}$ with an amplitude of $2-4 \mathrm{~mm}$. Following WBV or control, participants were assessed for vertical jump performance, MVIC and quadricep muscle soreness.

Participants returned to the laboratory 24, 48, and 72 hours following the EIMD. These sessions consisted of a pretest of vertical jump performance, MVIC EMG and muscle soreness in the quadriceps followed immediately by WBV treatment or control protocol identical to visit one. After treatment/control, all measures were reassessed.

\subsection{Reliability of the Measurements}

Three days of measurements were obtained in familiarization sessions. Measurement reliability and precision were quantified through the intraclass correlation coefficient (ICC) with a $95 \%$ confidence interval. The ICC value for vertical jump was $\mathrm{R}=0.92$.

\subsection{Data Analyses}

Five 2x8 (group $\mathrm{x}$ time) mixed factor analysis of variance (ANOVA) tests were used to determine changes in VJH, PPO, RFD, GRFz, and EMG ratio. Groups were WBV or control and time was Day0Pre, Day0Post, Day24Pre, Day24Post, Day48Pre, Day48Post, Day72Pre, and Day72 Post. If interactions occurred they were followed up with one-way ANOVAs while main effects were followed up with least significant difference (LSD) post-hoc analyses for pairwise differences. All analyses were conducted using SPSS software (SPSS 20, Inc., Chicago, IL). Statistical significance was determined as $\mathrm{P}<0.05$.

\section{Results}

\subsection{Vertical Jump}

For VJH or PPO, there were no significant ( $\mathrm{p}>0.05)$ interactions and no significant $(\mathrm{p}>0.05)$ main effects for group but there were significant $(\mathrm{p}<0.001)$ main effects for time. For VJH 0Pre was greater than all other time points and 72Pre was greater than 0Post, 24Pre, 24Post, 48Pre, 48Post, and 72Post (Figure 1). For PPO, 0Pre was greater than all other time points and 72Pre was greater than 24Pre, 24Post, 48Pre, 48Post, and 72Post (Table 1). 


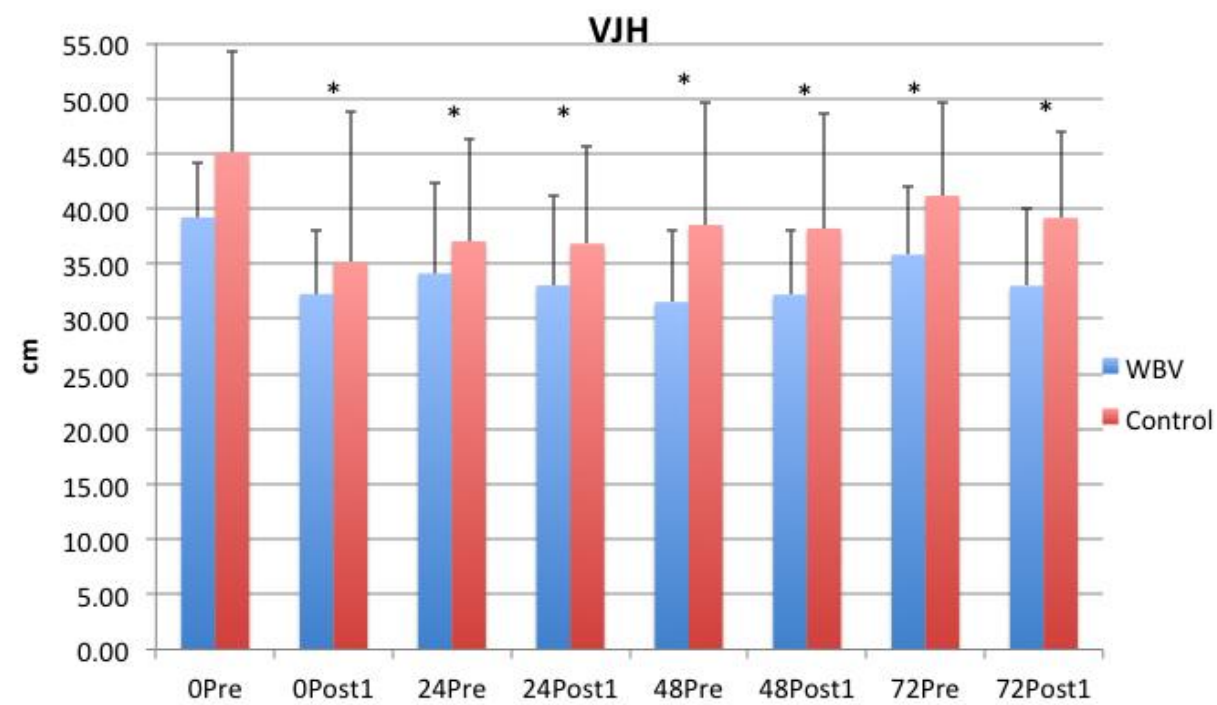

Figure 1. Vertical Jump Height

Figure 1. Means and standard deviations for VJH between groups and across time following exercise induced muscle damage. Significant $(\mathrm{p}<0.05)$ differences from 0Pre are indicated with *.

For GRFz, there was no significant ( $p>0.05)$ interaction or main effect for group but there was a significant $(\mathrm{p}<0.001)$ main effect for time. 0 Pre was greater than all other time points, 48Pre was greater than 72Post, and 72Pre was greater than 72Post (Table 1).

For RFD, there was no significant ( $p>0.05)$ interaction or main effect for group but there was a significant $(\mathrm{p}<0.001)$ main effect for time. 0Pre was greater than all other time points; 48Pre was less than 0Post, 24Pre, 24Post, 72Pre, and 72Post and 48Post were less than 72Pre and 72Post (Table 1).

\begin{tabular}{|c|c|c|c|c|c|c|c|c|c|c|c|c|c|c|c|c|}
\hline & \multicolumn{2}{|c|}{ OPre } & \multicolumn{2}{|c|}{ OPost1 } & \multicolumn{2}{|c|}{ 24Pre } & \multicolumn{2}{|c|}{ 24Post1 } & \multicolumn{2}{|c|}{ 48Pre } & \multicolumn{2}{|c|}{ 48Post1 } & \multicolumn{2}{|c|}{ 72Pre } & \multicolumn{2}{|c|}{ 72Post1 } \\
\hline & Mean & SD & Mean & SD & Mean & SD & Mean & SD & Mean & SD & Mean & SD & Mean & SD & Mean & SD \\
\hline \multicolumn{17}{|l|}{ PPO (W) } \\
\hline WBV & 2997.19 & 500.16 & 602.37 & 413.61 & 594.96 & 576.60 & 559.14 & 627.02 & 466.55 & 518.26 & 461.68 & 456.80 & 684.83 & 438.13 & 587.05 & 507.99 \\
\hline Control & 3313.78 & 765.62 & 881.87 & $969.76 *$ & 888.03 & $709.88^{*}$ & 841.21 & $700.66^{*}$ & 947.77 & $823.14^{*}$ & 951.14 & $825.93^{*}$ & 1131.02 & $685.02^{*}$ & 1056.15 & $621.33^{*}$ \\
\hline \multicolumn{17}{|l|}{ RFD (N/s) } \\
\hline WBV 3 & 35290.56 & 9910.29 & 28309.99 & 12587.37 * & 24571.26 & 11242.00 * & 27657.40 & 11484.02 & 19666.22 & 9922.31 & 23030.32 & 11882.35 & 26144.95 & 14340.48 * & 26360.40 & 12460.13 \\
\hline Control3 & 39707.88 & 18315.46 & 26290.53 & 13245.30 & 27829.14 & 14039.02 & 26628.89 & 15136.31 & 24044.13 & 12000.85 & 26705.55 & 15809.33 & 33087.62 & 14155.71 & 31392.48 & 12979.35 \\
\hline \multicolumn{17}{|c|}{ GRFz $(N)$} \\
\hline WBV & 239.21 & 27.72 & 219.63 & 29.18 * & 217.54 & 35.37 & 209.30 & 30.20 * & 220.99 & $31.48 *$ & 218.86 & $34.02 *$ & 219.63 & 20.81 * & 211.20 & $26.85 *$ \\
\hline Control & 251.87 & 36.57 & 224.18 & $28.01^{*}$ & 229.20 & 17.99 & 217.38 & 27.57 & 230.48 & 25.38 & 223.89 & 40.72 & 236.54 & $20.66^{*}$ & 225.23 & $18.83^{*}$ \\
\hline \multicolumn{17}{|l|}{ pEMG Ratio } \\
\hline WBV & 1.89 & 0.59 & 1.39 & 0.43 & 1.73 & 0.84 & 1.79 & 1.02 & 2.48 & 1.79 & 2.70 & 1.07 & 2.16 & 0.49 & 2.46 & 0.67 \\
\hline Control & 3.03 & 2.69 & 1.35 & 0.55 & 2.13 & 0.82 & 1.83 & 1.25 & 2.46 & 1.20 & 2.22 & 0.88 & 2.02 & 0.69 & 1.95 & 0.93 \\
\hline
\end{tabular}

\section{$3.2 E M G$}

For peak EMG ratio, there was no significant ( $\mathrm{p}>0.05)$ interaction or main effect for group was found but a significant $(\mathrm{p}<0.05)$ main effect for time was found. With 0Pre being greater than 0Post1 and 24Pre (Table 1).

\subsection{Visual Analog Scale}

For DOMS, there was no significant ( $p>0.05)$ interaction or main effect for group but there was a significant $(\mathrm{p}<0.001)$ main effect for time. 0Pre and 0Post were less than all time points. 24Pre were less than 48Pre, 48Post, 72Pre, and 72Post while 48Pre was greater than 72Pre and 72Post (Figure 2). 


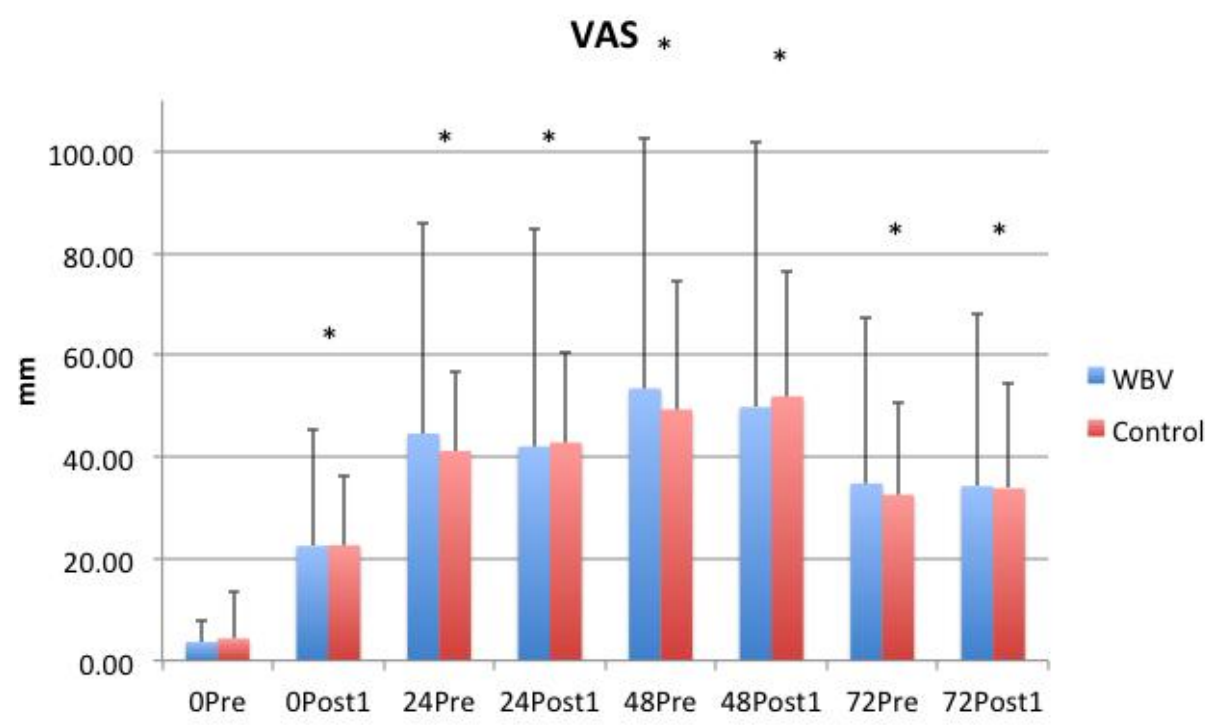

Figure 2. Visual Analogue Scale

Figure 2. Means and standard deviations for VAS between groups and across time following exercise induced muscle damage. Significant $(\mathrm{p}<0.05)$ differences from 0Pre are indicated with *.

\section{Discussion}

The aim of this investigation was to determine the effect of WBV on vertical jump performance following exercise induced muscle damage (EIMD). The EIMD protocol resulted in an immediate and prolonged detrimental effect on vertical jump performance; however, no differences were found between WBV and control groups. DOMS peaked at 48 hours post exercise, while VJH, PPO, RFD, GRFz, and peak EMG ratio all changed over time, indicative of decreased performance. To our knowledge, no previous research has investigated the effects of WBV on vertical jump performance following EIMD. Past research has either examined the effects of EIMD on vertical jump performance without WBV (Avela, Kyrolainen, Komi, \& Rama, 1999; Byrne \& Eston, 2002b), effects of WBV on vertical jump performance without EIMD (Adams et al., 2009; Bazett-Jones, Finch, \& Dugan, 2008; Bedient et al., 2009; Bosco et al., 1998; Bullock et al., 2008; Cochrane, Legg, \& Hooker, 2004; Cormie, Deane, Triplett, \& McBride, 2006; Dabbs, Munoz, Tran, Brown, \& Bottaro, 2011; Lamont et al.), or the effects of WBV on muscle recovery alone (Aminian-Far et al., 2011; Ayles, Graven-Nielsen, \& Gibson, 2011; Bakhtiary et al., 2007; Broadbent et al., 2008; Lau \& Nosaka, 2011; Rhea et al., 2009; Weerakkody et al., 2003). Therefore, this current study is novel in the performance and muscle recovery literature.

Previous research supports the current findings that vertical jump performance decreases immediately and up to 3 days after EIMD (Avela et al., 1999; Byrne \& Eston, 2002b), irrespective of WBV treatment. In Byrne \& Eston's investigation they found decreases in squat jump height, depth jump height and counter-movement jump height following damage and for 3 days after (Byrne \& Eston, 2002b). The present study extends their findings by measuring PPO, RFD and GRFz. Since our findings for PPO, RFD, and GRFz had similar decreases as VJH, it might be expected that these decreases would be similar in other jump performance studies. To our knowledge there are not any other studies that have looked at vertical jump PPO following EIMD. However, it has been shown that knee extensor PPO in isokinetic cycling (Sargeant \& Dolan, 1987) and a Wingate cycle test (Byrne \& Eston, 2002a) is decreased immediately following eccentric muscle actions and continues to be depressed up to 2 days post damage. A decrease in PPO has also been shown during intermittent cycle ergometer maximal sprints (10x6s) following 10 sets of 10 plyometric jumps to induce damage (Twist \& Eston, 2005). These results are similar to the current results. Since PPO is a critical variable in athletic performance, it is important to limit any reductions in lower-body PPO.

In the current study, we incorporated WBV as a recovery modality aimed at attenuating a reduction in performance but it was not successful as measured by VJH, PPO, RFD, and GRFz. Previous literature has shown mixed results when examining the effects of WBV on vertical jump performance. Some research has shown increases in VJH, PPO, RFD, and GRFz following WBV exposure (Adams et al., 2009; Bedient et al., 2009; Cormie et al., 2006; Dabbs et al., 2011), indicating a neuromuscular facilitation or potentiation effect. A recent study found increases in GRFz in recreationally trained individuals following WBV exposure compared to a control condition during a maximal vertical jump (Dabbs et al., 2011) while another study found no differences in isometric muscle RFD following WBV (Humphries, Warman, Purton, Doyle, \& Dugan, 2004). However, it appears that when muscle is damaged, it alters the effectiveness of WBV as a recovery modality. As previously mentioned, WBV has been examined as a recovery modality in the upper (Lau \& Nosaka, 2011) and lower extremities (Ayles et al., 2011; Broadbent et al., 2008; Rhea et al., 2009), when measuring pain, force production, and clinical variables but has not been investigated for vertical jump performance effects. These mixed results are most likely due to the use of varying damage protocols, vibration exposures, and extremities tested.

In this investigation, VM muscle activity was measured during the vertical jump via surface EMG and was calculated as a ratio of the VM peak EMG to the MVIC EMG. The ratio exhibited over time following muscle damage but no difference between groups. Previous research has investigated WBV and EMG RMS in the quadriceps while squatting 
and found increased activation following WBV compared to no vibration (Roelants, Verschueren, Delecluse, Levin, \& Stijnen, 2006) in non-damaged muscle. A study by Cormie et. al measured VJH and integrated EMG and found increases in VJH immediately following WBV exposure when compared to no vibration but no differences in integrated EMG (Cormie et al., 2006), in non-damaged muscle. Our findings are similar to Cormie et. al, in regards to no group differences in muscle activity, however they did not induce muscle damage. Since EIMD changes the contractile properties of muscle (Armstrong, 1990), this may account for differences between our studies. Our findings suggest that WBV has a detrimental effect on vertical jump peak normalized muscle activity of the VM.

It is necessary to discuss how the potentiating mechanism of the stretch shortening cycle attenuates the detrimental vertical jump performance effects following EIMD. It has been suggested that excitation-contraction coupling is impaired following muscle damage (Edwards, Hill, Jones, \& Merton, 1977), decreasing the release of calcium per action potential (Warren, Ingalls, Lowe, \& Armstrong, 2001), leading to an inability to activate force-generating structures. It has been proposed that after EIMD, a reduction in stretch reflex sensitivity and muscle stiffness occur (Horita, Komi, Nicol, \& Kyrolainen, 1996; Nicol, Komi, Horita, Kyrolainen, \& Takala, 1996), leading to decreased force potentiating mechanisms. It has also been suggested that EIMD results in modifications in pre-landing motor control, possibly brought on by central inhibition due to muscle soreness (Horita et al., 1996). Since the stretch shortening cycle is a key component in a countermovement vertical jump, this may help explain our findings of decreases in VJH, PPO, RFD, and GRFz following EIMD.

In conclusion, it appears that WBV has no positive effect on VJH, PPO, RFD, GRFz, or VM peak EMG ratio following EIMD. Therefore, utilizing WBV as a recovery modality is ineffective. Future research should investigate the effects of a variety of WBV exposure times, frequencies, amplitudes, and rest intervals on performance and following EIMD. Additionally, different levels of soreness should be examined to determine if the amount of soreness affects WBV as a recovery modality. Finally, trained athletes and males should also be examined with similar protocols to determine effects in different participant populations.

\section{References}

Adams, J. B., Edwards, D., Serviette, D., Bedient, A. M., Huntsman, E., Jacobs, K. A., . . . Signorile, J. F. (2009). Optimal frequency, displacement, duration, and recovery patterns to maximize power output following acute wholebody vibration. $J$ Strength Cond Res, 23(1), 237-245. doi: 10.1519/JSC.0b013e3181876830

Aminian-Far, A., Hadian, M. R., Olyaei, G., Talebian, S., \& Bakhtiary, A. H. (2011). Whole-body vibration and the prevention and treatment of delayed-onset muscle soreness. J Athl Train, 46(1), 43-49. doi: 10.4085/1062-6050-46.1.43

Armstrong, R. B. (1990). Initial events in exercise-induced muscular injury. Med Sci Sports Exerc, 22(4), 429-435.

Armstrong, R. B., Warren, G. L., \& Warren, J. A. (1991). Mechanisms of exercise-induced muscle fibre injury. Sports Med, 12(3), 184-207.

Avela, J., \& Komi, P. V. (1998). Reduced stretch reflex sensitivity and muscle stiffness after long-lasting stretchshortening cycle exercise in humans. Eur J Appl Physiol Occup Physiol, 78(5), 403-410.

Avela, J., Kyrolainen, H., Komi, P. V., \& Rama, D. (1999). Reduced reflex sensitivity persists several days after longlasting stretch-shortening cycle exercise. J Appl Physiol, 86(4), 1292-1300.

Ayles, S., Graven-Nielsen, T., \& Gibson, W. (2011). Vibration-induced afferent activity augments delayed onset muscle allodynia. J Pain, 12(8), 884-891. doi: S1526-5900(11)00476-7 [pii] 10.1016/j.jpain.2011.02.355

Bakhtiary, A. H., Safavi-Farokhi, Z., \& Aminian-Far, A. (2007). Influence of vibration on delayed onset of muscle soreness following eccentric exercise. $\mathrm{Br} J$ Sports Med, 41(3), 145-148. doi: bjsm.2006.031278 [pii] 10.1136/bjsm.2006.031278

Bazett-Jones, D., Finch, H., \& Dugan, E. (2008). Comparing the effects of various whole-body vibration accelerations on countermovement jump perfomance. J Sci Med Sport, 7, 144-150.

Bedient, A. M., Adams, J. B., Edwards, D., Serviette, D., Huntsman, E., Mow, S. E., . . . Signorile, J. F. (2009). Displacement and frequency for maximizing power output resulting from a bout of whole-body vibration. $J$ Strength Cond Res, 23(6), 1683-1687.

Bosco, C., Cardinale, M., Tsarpela, O., Colli, R., Tihanyi, J., VonDuvillard, S., \& Viru, A. (1998). The influence of whole body vibration on jumping performance. Bio Sports, 15, 157-164.

Broadbent, S., Rousseau, J. J., Thorp, R. M., Choate, S. L., Jackson, F. S., \& Rowlands, D. S. (2008). Vibration therapy reduces plasma IL6 and muscle soreness after downhill running. Br J Sports Med, 44(12), 888-894. doi: bjsm.2008.052100 [pii]10.1136/bjsm.2008.052100

Bullock, N., Martin, D. T., Ross, A., Rosemond, C. D., Jordan, M. J., \& Marino, F. E. (2008). Acute effect of wholebody vibration on sprint and jumping performance in elite skeleton athletes. J Strength Cond Res, 22(4), 1371-1374. doi: 10.1519/JSC.0b013e31816a44b5

Byrne, C., \& Eston, R. (2002a). Maximal-intensity isometric and dynamic exercise performance after eccentric muscle actions. J Sports Sci, 20(12), 951-959. doi: 10.1080/026404102321011706

Byrne, C., \& Eston, R. (2002b). The effect of exercise-induced muscle damage on isometric and dynamic knee extensor strength and vertical jump performance. J Sports Sci, 20(5), 417-425. doi: 10.1080/026404102317366672 
Cheung, K., Hume, P., \& Maxwell, L. (2003). Delayed onset muscle soreness : treatment strategies and performance factors. Sports Med, 33(2), 145-164. doi: 3325 [pii]

Cochrane, D. J., Legg, S. J., \& Hooker, M. J. (2004). The short-term effect of whole-body vibration training on vertical jump, sprint, and agility performance. J Strength Cond Res, 18(4), 828-832. doi: 14213 [pii] 10.1519/14213.1

Connolly, D. A., Sayers, S. P., \& McHugh, M. P. (2003). Treatment and prevention of delayed onset muscle soreness. $J$ Strength Cond Res, 17(1), 197-208.

Cormie, P., Deane, R. S., Triplett, N. T., \& McBride, J. M. (2006). Acute effects of whole-body vibration on muscle activity, strength, and power. J Strength Cond Res, 20(2), 257-261. doi: R-17835 [pii] 10.1519/R-17835.1

Dabbs, N. C., Munoz, C. X., Tran, T. T., Brown, L. E., \& Bottaro, M. (2011). Effect of different rest intervals after whole-body vibration on vertical jump performance. $J$ Strength Cond Res, 25(3), 662-667. doi: 10.1519/JSC.0b013e318207eafc

Edwards, R., Hill, D., Jones, D., \& Merton, P. (1977). Fatigue of Long Duration in Human Skeletal Muscle After Exercise. . Journal of Physiology, 272, 769-778.

Friden, J., Sjostrom, M., \& Ekblom, B. (1983). Myofibrillar damage following intense eccentric exercise in man. Int $J$ Sports Med, 4(3), 170-176.

Gibson, W., Arendt-Nielsen, L., \& Graven-Nielsen, T. (2006). Delayed onset muscle soreness at tendon-bone junction and muscle tissue is associated with facilitated referred pain. Exp Brain Res, 174(2), 351-360. doi: 10.1007/s00221-0060466-y

Horita, T., Komi, P. V., Nicol, C., \& Kyrolainen, H. (1996). Stretch shortening cycle fatigue: interactions among joint stiffness, reflex, and muscle mechanical performance in the drop jump [corrected]. Eur J Appl Physiol Occup Physiol, 73(5), 393-403.

Horita, T., Komi, P. V., Nicol, C., \& Kyrolainen, H. (1999). Effect of exhausting stretch-shortening cycle exercise on the time course of mechanical behaviour in the drop jump: possible role of muscle damage. Eur J Appl Physiol Occup Physiol, 79(2), 160-167.

Humphries, B., Warman, G. E., Purton, J., Doyle, T. L. A., \& Dugan, E. (2004). The Influence of Vibration on Muscle Activation and Rate of Force Development during Maximal Isometric Contractions. Journal of Sports Science and Medicine, 3, 16-22.

Lamont, H. S., Cramer, J. T., Bemben, D. A., Shehab, R. L., Anderson, M. A., \& Bemben, M. G. The acute effect of whole-body low-frequency vibration on countermovement vertical jump performance in college-aged men. $J$ Strength Cond Res, 24(12), 3433-3442. doi: 10.1519/JSC.0b013e3181c1ff7e

Lau, W. Y., \& Nosaka, K. (2011). Effect of vibration treatment on symptoms associated with eccentric exercise-induced muscle damage. Am J Phys Med Rehabil, 90(8), 648-657. doi: 10.1097/PHM.0b013e3182063ac8

McHugh, M. P., Connolly, D. A., Eston, R. G., \& Gleim, G. W. (1999). Exercise-induced muscle damage and potential mechanisms for the repeated bout effect. Sports Med, 27(3), 157-170.

Nicol, C., Komi, P. V., Horita, T., Kyrolainen, H., \& Takala, T. E. (1996). Reduced stretch-reflex sensitivity after exhausting stretch-shortening cycle exercise. Eur J Appl Physiol Occup Physiol, 72(5-6), 401-409.

Proske, U., \& Morgan, D. L. (2001). Muscle damage from eccentric exercise: mechanism, mechanical signs, adaptation and clinical applications. J Physiol, 537(Pt 2), 333-345. doi: PHY_13112 [pii]

Rhea, M. R., Bunker, D., Marin, P. J., \& Lunt, K. (2009). Effect of iTonic whole-body vibration on delayed-onset muscle soreness among untrained individuals. $J$ Strength Cond Res, 23(6), 1677-1682. doi: 10.1519/JSC.0b013e3181b3f6cd

Roelants, M., Verschueren, S. M., Delecluse, C., Levin, O., \& Stijnen, V. (2006). Whole-body-vibration-induced increase in leg muscle activity during different squat exercises. J Strength Cond Res, 20(1), 124-129. doi: R-16674 [pii]10.1519/R-16674.1

Sargeant, A. J., \& Dolan, P. (1987). Human muscle function following prolonged eccentric exercise. Eur J Appl Physiol Occup Physiol, 56(6), 704-711.

Sayers, S. P., Harackiewicz, D. V., Harman, E. A., Frykman, P. N., \& Rosenstein, M. T. (1999). Cross-validation of three jump power equations. Med Sci Sports Exerc, 31(4), 572-577.

Sayers, S. P., Knight, C. A., Clarkson, P. M., Van Wegen, E. H., \& Kamen, G. (2001). Effect of ketoprofen on muscle function and sEMG activity after eccentric exercise. Med Sci Sports Exerc, 33(5), 702-710.

Schutzer, K. A., \& Graves, B. S. (2004). Barriers and motivations to exercise in older adults. Prev Med, 39(5), 10561061. doi: S0091743504002026 [pii] 10.1016/j.ypmed.2004.04.003

Stauber, W. T., Clarkson, P. M., Fritz, V. K., \& Evans, W. J. (1990). Extracellular matrix disruption and pain after eccentric muscle action. J Appl Physiol, 69(3), 868-874.

Twist, C., \& Eston, R. (2005). The effects of exercise-induced muscle damage on maximal intensity intermittent exercise performance. Eur J Appl Physiol, 94(5-6), 652-658. doi: 10.1007/s00421-005-1357-9 
Warren, G. L., Ingalls, C. P., Lowe, D. A., \& Armstrong, R. B. (2001). Excitation-contraction uncoupling: major role in contraction-induced muscle injury. Exerc Sport Sci Rev, 29(2), 82-87.

Weerakkody, N. S., Percival, P., Hickey, M. W., Morgan, D. L., Gregory, J. E., Canny, B. J., \& Proske, U. (2003). Effects of local pressure and vibration on muscle pain from eccentric exercise and hypertonic saline. Pain, 105(3), 425435. doi: S0304395903002574 [pii]. 